\title{
Soft X-ray Spectromicroscopy at the Canadian Light Source
}

Jian Wang ${ }^{1}$, Jan Geilhufe ${ }^{1}$, Yingshen Lu ${ }^{1}$, James J. Dynes ${ }^{1}$, Jigang Zhou ${ }^{1}$, Russ Berg ${ }^{1}$, Adam F.G. Leontowich $^{1}$, Ian Coulthard ${ }^{1}$, John Swirsky ${ }^{1}$, Chithra Karunakaran ${ }^{1}$, Adam P. Hitchcock ${ }^{2}$ and Stephen G. Urquhart ${ }^{3}$

1. Canadian Light Source Inc., Saskatoon, Canada

2. Brockhouse Institute for Materials Research, McMaster University, Hamilton, Canada

3. Department of Chemistry, University of Saskatchewan, Saskatoon, Canada

* Corresponding author, jian.wang@lightsource.ca

The soft X-ray Spectro-Microscopy (SM) beamline at the Canadian Light Source (CLS) [1] consists of an APPLE II type Elliptically Polarizing Undulator (EPU, arbitrary polarizations), a plane grating monochromator (PGM, 130 - $2700 \mathrm{eV}$ ), focusing optics, and three endstations, i.e. an ambient Scanning Transmission X-ray Microscope (STXM), a Cryo-STXM microscope and an X-ray Photoemission Electron Microscope (X-PEEM), as shown in Fig. 1. Both STXMs and X-PEEM can be used to measure morphology/structure, quantitative distributions (maps) of chemical components in 2D (3D in STXM), molecular orientation, as well as obtain the electronic structure of materials via spatially-resolved Near Edge X-ray Absorption Fine Structure (NEXAFS) spectroscopy. Since 2007 this facility has been used to study samples from the fields of materials sciences, environmental/earth sciences and life sciences. All three spectro-microscopes achieve high spatial resolution (sub $30 \mathrm{~nm}$ ), high spectral resolution $(<0.05 \mathrm{eV})$, and can be used with a wide range of samples, including in situ and operando studies.

Ambient-STXM (Fig. 1b), provides high quality 2D/3D chemical and morphological imaging and sub$10 \mathrm{~nm}$ spatial resolution by STXM-ptychography. Dry and wet samples can be measured. Devices are available for in situ azimuthal and polar sample rotation; imposing magnetic fields up to $5 \mathrm{kG}$ at the center of a permanent dipole magnet $(\sim 3 \mathrm{kG}$ at the edge); measuring samples with variable cooling/heating $\left(5-80{ }^{\circ} \mathrm{C}\right)$, humidity, and potential. In addition, advanced STXM setups, such as low energy X-ray fluorescence (LEXRF) detection using silicon drift detectors (SDD), total electron yield (TEY) detection through sample current or channeltron, and coherent diffractive imaging (CDI), i.e. ptychography, using a CCD, are routinely accessible. An offline Olympus BX51 optical microscope can be used to image and record sample position to assist rapid sample navigation in STXM.

Cryo-STXM (Fig. 1c), provides spectromicroscopy of samples at cryogenic temperatures (below -170 ${ }^{\circ} \mathrm{C}$ ) and ultra-high vacuum (UHV) conditions ( $<10^{-8}$ Torr). It is able to conduct cryo-tomography measurements using a JEOL 2010 FasTEM Goniometer and a Gatan 630 high-tilt cryo-sample holder with sample rotation from $-70^{\circ}$ to $+70^{\circ}$ under UHV conditions. Samples on 3-pin kinematic STXM sample plates, the same as used in ambient-STXM, can also be studied (Fig. 1d).

X-PEEM (Fig. 1e) is a commercial Elmitec microscope capable of better than $50 \mathrm{~nm}$ spatial resolution on UHV compatible, flat and conductive sample surfaces. The instrument can be configured as an energy unfiltered X-PEEM to measure organic materials or other low conductivity samples. It can also be configured as an energy filtered X-PEEM (Fig. 1f) in order to enable X-ray Photoelectron Spectroscopy (XPS). The X-PEEM chamber is attached to a UHV preparation chamber which includes several evaporation ports, a quartz crystal micro balance, ion sputtering and a LEED/Auger system. An offline Cressington 208HR high resolution sputter coater can be used to coat insulating samples with 0.1 
nm of metal (Pt, Pd, Cr, Au, etc.).

The CLS SM beamline is currently part way through a major upgrade with the newly commissioned Cryo-STXM as the first subproject. A new PGM monochromater is under design with the goals to improve the beamline flux performance above 1800 up to $\sim 3500 \mathrm{eV}$ using multilayered grating/mirror technology, and to enhance mechanical stability and cooling. Finally the X-PEEM system will be upgraded to improve spot size by converting from horizontal to vertical column orientation and implementing new refocusing optics. In addition a switching system will be implemented to allow rapid change between energy unfiltered and filtered X-PEEM $[2,3]$.

References:

[1] K.V. Kaznatcheev, C. Karunakaran, U.D. Lanke, S.G. Urquhart, M. Obst, A.P. Hitchcock, Nucl. Instr. Meth. Phys. Res. A 582 (2007) 96-99.

[2] CLS-SM publications: https://sm.lightsource.ca/publications/

[3] CLS is funded by the Canada Foundation for Innovation, the Natural Sciences and Engineering Research Council of Canada, the National Research Council Canada, the Canadian Institutes of Health Research, the Government of Saskatchewan, Western Economic Diversification Canada, and the University of Saskatchewan.
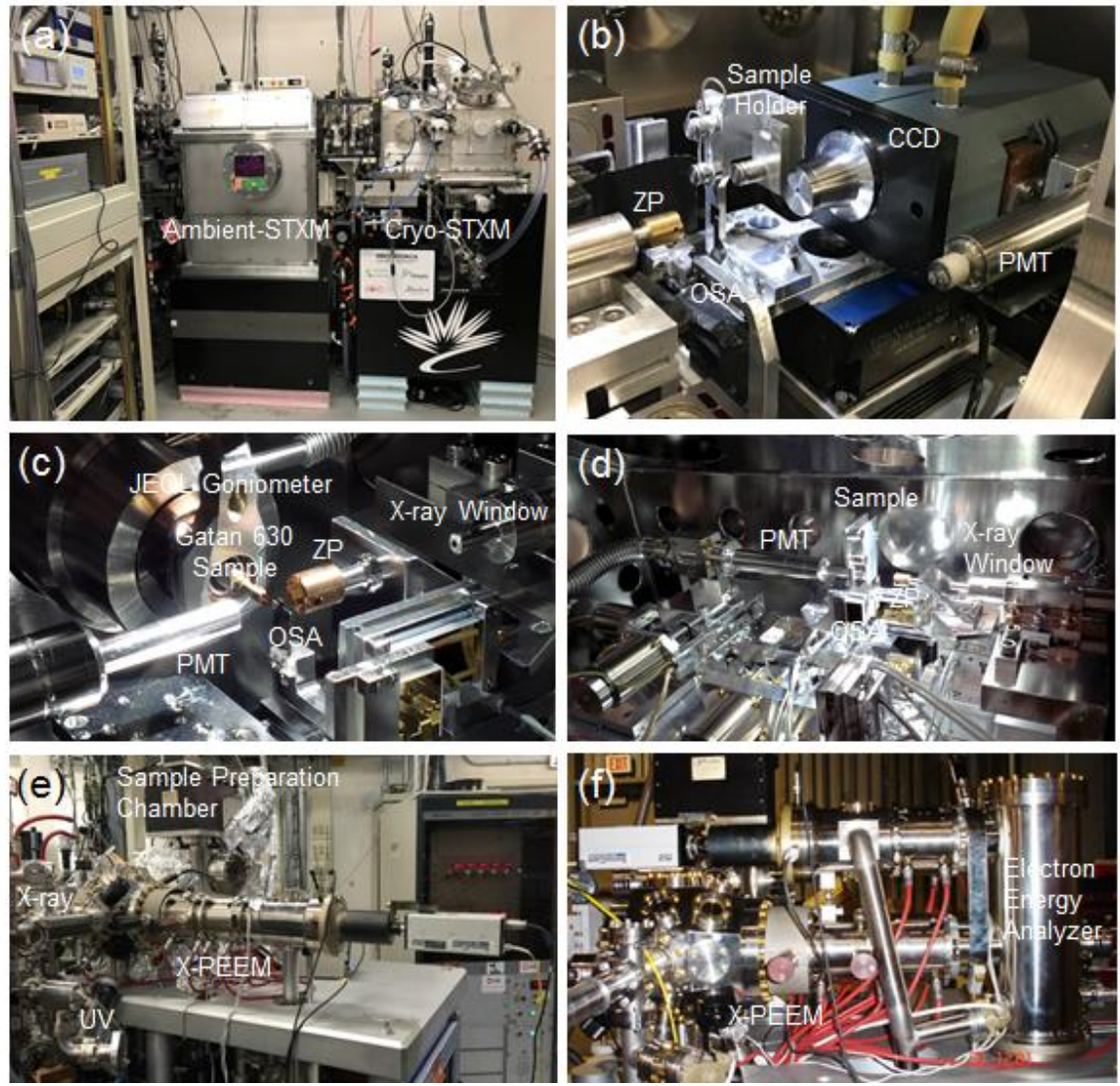

Figure 1. (a) Photograph of the CLS Ambient-STXM and Cryo-STXM; (b) Inside view of the Ambient-STXM; (c) Inside view of the Cryo-STXM cryo-tomography mode; (d) Inside view of the Cryo-STXM conventional mode; (e) Photograph of the CLS energy unfiltered X-PEEM; (f) Photograph of the energy filtered X-PEEM. 